\title{
Dilemas na regulação do acesso à atenção especializada de crianças com condições crônicas complexas de saúde
}

\author{
Dilemmas in regulating access to specialized care for children with \\ complex chronic health conditions
}

Michele Ferreira da Silva (https://orcid.org/0000-0003-0630-7436) ${ }^{1}$

Martha Cristina Nunes Moreira (https://orcid.org/0000-0002-7199-3797) ${ }^{2}$

${ }^{1}$ Instituto de Puericultura e Pediatria Martagão Gesteira, Universidade Federal do Rio de Janeiro. R. Bruno Lobo 50, Cidade Universitária. 21941-912 Rio de Janeiro RJ Brasil.

michelefsilva@ippmg.ufrj.br

${ }^{2}$ Instituto Fernandes

Figueira, Fundação Oswaldo

Cruz. Rio de Janeiro RJ

Brasil.

\begin{abstract}
The scope of this article was to analyze the access of children with complex chronic health conditions to specialized care, identifying their strengths and weaknesses from the perspective of speech therapists. The selection of research subjects was made considering the participation in a virtual group whose dynamics signaled the difficulty of members, municipal civil servants, in the conduct of referring patients to health services. Face-to-face interviews were conducted with 14 speech therapists from this group who work with children. The study of the difficulties faced by the interviewees made it possible to identify that care via the Regulation System is an aggravating factor in difficulties of access. In this respect, the problems mentioned were grouped into three topics: (1) Waiting time for making appointments; (2) Distance from the care centers; and (3) Misconceptions in the referral process. In addition, the work of the Expanded Family Health and Primary Care Service was identified as a relevant factor in facilitating children's access to specialized care services in partnership with regulation. The conclusion drawn is that the presence of mediators in health care benefits the population with difficulty of access to services, including children with complex chronic health conditions.
\end{abstract}

Key words Child, Chronic disease, Access to health services, Speech therapy
Resumo O presente artigo objetivou analisar o acesso de crianças com condições crônicas complexas de saúde à atenção especializada, identificando seus dilemas e contrapontos. A seleção dos sujeitos de pesquisa foi realizada entre junho e setembro de 2017 e considerou a participação de fonoaudiólogos em um grupo virtual cuja dinâmica sinalizava a dificuldade dos integrantes, servidores públicos municipais, na conduta de encaminhar pacientes aos serviços de saúde. Foram realizadas entrevistas presenciais com 14 fonoaudiólogos deste grupo, que atuam com crianças. $O$ estudo das dificuldades enfrentadas pelos entrevistados possibilitou identificar que a regulação assistencial via SISREG é um agravante das difculdades de acesso. Neste sentido, os dilemas mencionados foram agrupados em três eixos temáticos: espera para marcação das consultas, distância dos locais de atendimento e equívocos no processo de encaminhamento. Além disso, a atuação do Núcleo Ampliado de Saúde da Família e Atenção Básica (NASF-AB) foi identificada como um fator relevante na facilitação do acesso de crianças aos serviços da atenção especializada no trabalho em parceria com a regulação. Conclui-se que a presença de mediadores no âmbito dos cuidados em saúde beneficia a população com dificuldades de acesso aos serviços, dentre as quais, as crianças com condições crônicas complexas de saúde.

Palavras-chave Criança, Doenças crônicas, Acesso aos serviços de saúde, Fonoaudiologia 


\section{Introdução}

Um dos mecanismos para organizar o acesso e o fluxo das crianças com condições crônicas complexas aos serviços de saúde na atenção especializada é a regulação assistencial. Regulação é um termo que apresenta concepções variadas. Segundo Vilarins et al. ${ }^{1}$ existem três níveis de regulação no que se refere a atuação do Estado no setor saúde: regulação sobre os sistemas de saúde, regulação da atenção à saúde e regulação do acesso à assistência. Este último nível envolve as estratégias do SUS que buscam organizar as centrais de regulação, a fim de direcionar o planejamento e a execução das ações que visam facilitar $\mathrm{o}$ acesso da população aos serviços de saúde ${ }^{2,3}$. O Sistema Nacional de Regulação (SISREG) é um sistema de informação em saúde elaborado pelo DATASUS no formato de plataforma online, disponibilizado pelo governo federal para os municípios a fim de instrumentalizar a regulação em saúde no Brasil ${ }^{3}$.

O presente artigo considera a dificuldade de acesso de crianças com condições crônicas complexas de saúde aos serviços de saúde especializados e parte da constatação de dois grandes problemas: 1) os profissionais identificam e diagnosticam os problemas de saúde e/ou desenvolvimento, e recomendam um encaminhamento para serviços especializados; 2) as famílias recebem esses encaminhamentos e se veem diante da busca por acesso, que se torna uma "peregrinação terapêutica", no sentido que o itinerário dessa busca por cuidados se vê obstaculizado pelas dificuldades de acesso ${ }^{10}$.

As crianças com condições crônicas complexas de saúde demandam uma linha de cuidados que incorpore ações de estimulação essencial e reabilitação ${ }^{5,6}$. Essas crianças podem se tornar frequentadoras destes espaços considerando que o tratamento da doença e das alterações no processo de desenvolvimento resultantes da condição de adoecimento é contínuo e de longa duração. $\mathrm{Na}$ infância, devido à intensa neuroplasticidade, ocorre o período de maior desenvolvimento, crescimento e aprendizagem na vida do indivíduo, e por isso é o momento mais oportuno para a realização de estimulação e reabilitação. Logo, torna-se imprescindível que o tratamento dessas crianças seja iniciado nos serviços da atenção especializada imediatamente após a identificação de alterações no desenvolvimento ${ }^{7-9}$.

A Fonoaudiologia é uma das áreas da saúde que se insere nessa linha de cuidados integral voltada à criança nos três níveis de atenção à saúde (atenção primária, secundária e terciária). O cuidado fonoaudiológico de crianças em condições crônicas complexas de saúde na atenção especializada, em uma perspectiva multiprofissional e interdisciplinar, torna-se essencial para a prevenção dos distúrbios da comunicação humana e promoção da saúde neste grupo de crianças ${ }^{11,12}$.

Assim como proposto por Madureira et al. ${ }^{13}$, apoiar análises na perspectiva de profissionais sobre problemas referentes à emergência de demandas dirigidas por essa população se mostra relevante. Estes autores exploraram a perspectiva de fisioterapeutas sobre a sua própria atuação em um cenário de mudanças em que se identifica o aumento de crianças cronicamente adoecidas. Com base neste estudo, é possível afirmar que conversar com profissionais de uma área específica não significa restringir análises, mas apoiá-los em problemas concretos e produzir diagnósticos de problemas recentes.

O objetivo deste artigo é analisar o acesso de crianças em condições crônicas complexas de saúde à atenção especializada identificando dilemas e contrapontos na perspectiva de profissionais de fonoaudiologia à luz de uma análise sobre redes e mediadores.

\section{Metodologia}

Este artigo é fruto de uma dissertação de mestrado $^{14}$ que buscou compreender qual o alcance da dinâmica de um grupo virtual em discussões públicas sobre acesso, no campo da atenção integral, a crianças com condições crônicas complexas de saúde. Trata-se de uma pesquisa qualitativa aprovada pelo Comitê de Ética em Pesquisa. A coleta de dados foi realizada no período de junho a setembro de 2017 e contemplou a realização de entrevistas presenciais e individuais com roteiro semiestruturado referentes aos objetivos da pesquisa. Foram entrevistados 14 fonoaudiólogos, membros de um grupo virtual formado por profissionais desta categoria, todos servidores públicos municipais de um mesmo município.

A reunião de profissionais em grupos virtuais, relacionados às suas práticas de trabalho, vem se tornando uma tendência contemporânea. Reconhecendo essas novas articulações, que ultrapassam as fronteiras de serviços, a pesquisa teve acesso a um grupo constituído por fonoaudiólogos lotados em um município do estado do Rio de Janeiro, para a partir desse espaço convidar seus participantes para entrevistas presenciais. Ou seja, o grupo foi o universo de relações em 
que acessamos os participantes da pesquisa. $\mathrm{O}$ universo de relações nesse caso se apoia na busca do espaço virtual por profissionais da saúde como um lugar para encontros, trocas e busca de apoio social no intuito de responder aos problemas coletivamente enfrentados. Segundo Iriart e Silva ${ }^{15}$, as tecnologias de informação não só se tornaram formas facilitadas de acessar informações, mas também um rico ambiente para produção de subjetividades e de interação social. Acompanhando a atual tendência de expansão da comunicação virtual, as pesquisas sociais têm explorado esses novos meios de comunicação também como campos de pesquisa ${ }^{15-17}$.

Dos 14 entrevistados, 13 mulheres e um homem, todos com idade acima de 30 anos com trajetória profissional acima de 10 anos, e atuando com crianças em unidades de saúde do município, dentre os quais: Núcleo Ampliado de Saúde da Família e Atenção Básica (NASF-AB), Núcleo de Atenção Interdisciplinar do Desenvolvimento Infantil (NAIDI), Posto de Saúde (PS), Centro Municipal de Saúde (CMS), Centro Especializado de Reabilitação (CER) e maternidades. A fim de preservar o anonimato, os participantes receberam nomes fictícios referentes a sentimentos.

Sobre a seleção dos sujeitos de pesquisa, é importante pontuar algumas considerações: 1) embora uma das pesquisadoras seja fonoaudióloga, o estudo não tinha como objetivo de pesquisar exclusivamente fonoaudiólogos. O nosso interesse primordial era entender as dificuldades do acesso de crianças com condição crônica complexa de saúde aos serviços especializados, sem o interesse específico em uma determinada área assistencial. $\mathrm{O}$ acesso ao grupo virtual de fonoaudiologia se deu por indicação de uma informante privilegiada, que também é fonoaudióloga, integrante do grupo virtual e que pertence à rede de relações da pesquisadora; 2) o grupo virtual do qual participam os sujeitos entrevistados apresenta uma característica intrigante e peculiar. Uma de suas funções é buscar soluções para problemas relativos a referências, encaminhamentos e apoios que podem produzir acesso aos serviços de saúde de pessoas nas diversas faixas etárias. Interessou-nos entrevistar os fonoaudiólogos participantes deste grupo que trabalham com crianças, entendendo que o grupo tem uma ligação estreita com a produção de soluções para os problemas de acesso dessa população.

A primeira entrevista foi realizada com a administradora do grupo virtual, que indicou outros participantes para as entrevistas. Estes, por sua vez, indicaram outros, e assim, sucessivamen- te até que chegamos ao número de 14 entrevistas. $\mathrm{O}$ convite para os membros indicados ocorreu através de contato via aplicativo de mensagens, com número telefônico previamente informado pelos participantes que realizaram as indicações.

As entrevistas presenciais ocorreram em locais públicos, gravadas e transcritas pela própria pesquisadora e armazenadas para valorizar a confidencialidade das informações. As perguntas norteadoras da entrevista contemplaram questionamentos sobre a atuação destes profissionais na assistência de crianças em condições crônicas complexas de saúde sobre a experiência com o acesso das crianças aos serviços de saúde especializados, bem como sobre os propósitos de participação no grupo virtual.

A análise interna do material contemplou três etapas: 1) transcrições das falas na íntegra e a ordenação dos dados registrados em diário de campo; 2) avaliação da qualidade do material organizado a fim de verificar se as informações estavam completas; 3 ) elaboração de propostas de categorização a partir de releituras do material e da construção teórica do estudo ${ }^{18}$.

Em seguida, também foi realizada a leitura compreensiva do material selecionado e a construção de inferências a partir das transcrições das falas e registros de observação. Nesta fase foi importante efetuar a quebra de cada uma das ideias identificadas no material disponível e separá-las em categorias de análise, além de buscar as informações que ali estavam subentendidas. Por fim, buscou-se a elaboração da síntese com a construção criativa de possíveis significados ${ }^{18}$.

A análise dos dados deu-se à luz da teoria relacional das redes sociais ${ }^{19-22}$ que valoriza as interações e redes de relações promotoras de significados sobre problemas do cotidiano que, neste caso, se desenrolam em torno das necessidades de encaminhar e resolver as demandas de saúde e reabilitação de crianças com condições crônicas complexas de saúde. As entrevistas foram analisadas considerando-se as interações mediadas pelo significado atribuído nas relações com o cuidado e nas preocupações com o acesso de crianças à rede especializada.

\section{Resultados e discussão}

Esta discussão se organiza nos três eixos dos dilemas na regulação de vagas de atendimento via SISREG para crianças com condições crônicas complexas de saúde na rede de atenção especializada, fruto da análise empreendida pela pesquisa: 
1) espera para marcação das consultas; 2) distância dos locais de atendimento e 3 ) equívocos no processo de encaminhamento. Neste último eixo também é discutido o papel do Núcleo Ampliado de Saúde da Família e Atenção Básica (NASF-AB) no apoio aos profissionais reguladores situados na atenção primária no sentido de auxiliar o direcionamento do fluxo de encaminhamentos das crianças à atenção especializada.

\section{A espera pela marcação de consultas}

Nas entrevistas, alguns motivos foram mencionados a respeito de retardamento dos agendamentos para consultas e exames através do SISREG nas diferentes etapas do processo regulatório, dentre eles, a dificuldade para efetuar o agendamento da consulta e o tempo prolongado entre a marcação e a realização do atendimento.

Então assim, eu tinha uma marcação mensal, onde eu via que os meus pacientes não faltavam. (...) Tinha paciente que ia marcar hoje e o exame era amanhã. (...) Hoje o meu paciente chega aqui dizendo (...) que tá há um ano tentando marcar e eu sei que o meu tempo de marcação não é um ano. (...) Às vezes o procedimento demora muito tempo pra marcar, o paciente esquece. (Esperança, fonoaudióloga audiologista)

Nas unidades da atenção básica os médicos reguladores são os responsáveis por cadastrar os encaminhamentos via SISREG e realizar a coordenação do fluxo dos usuários pelos pontos de atenção à saúde. No entanto, prevalece a ausência de padronização desse processo de cadastro e de controle dos fluxos na rede. Desta forma, existem unidades que realizam as marcações durante ou logo após as consultas enquanto que em outras, as marcações estão sujeitas à disponibilidade de tempo do médico regulador da unidade.

$\mathrm{O}$ "regulador de vagas" é alguém que não necessariamente conhece ou acumula experiências no trabalho com as especificidades e necessidades de crianças crônicas complexas. Ou seja, ao serem encaminhadas, as crianças partem de alguém que realizou um diagnóstico - um tempo de espera ou barreiras de acesso podem incrementar atrasos no desenvolvimento -, na construção de uma linha de cuidado promotora de saúde. O regulador não interagiu com essa realidade e precisa ser sensibilizado com conhecimentos necessários para fazer esse sistema regulatório operar de forma a gerar benefícios e não iatrogêneses.

Nessa direção, com base em Barnes ${ }^{19}$, refletese sobre como o processo político encontra-se dependente de mediadores, de símbolos de con- fiança, de solidariedade e troca, que evoquem as dimensões interacionais e das redes de sociabilidade que dão sentido ao cuidado. A figura do "regulador de vagas" que desconhece as crianças, e não interage e nem participa dos seus cuidados, o deixa distante das necessidades e urgências que movimentam o cuidado às crianças com condições crônicas complexas de saúde que os profissionais entrevistados tão bem conhecem.

Outra questão referida foi a oferta limitada de vagas disponibilizadas pelos serviços especializados das unidades prestadoras. Alguns entrevistados revelaram que a espera pode ser bastante longa dependendo da especialidade. Os relatos não se referiram apenas a serviços de fonoaudiologia, mas também a outras especialidades que compõem a rede assistencial especializada. Foram citadas as especialidades médicas de Neurologia, Oftalmologia e Otorrinolaringologia. Segundo Cavalcanti et al. ${ }^{23}$, a dificuldade mais referida nos estudos sobre regulação em saúde é a limitada oferta de consultas e exames na rede assistencial que está diretamente relacionada às ações políticas das secretarias de saúde. Nas entrevistas, essa demora foi atribuída à escassez de profissionais e de unidades de saúde para atender a demanda da população.

O aumento de vagas ociosas também foi apontado como resultante da chegada do SISREG, seja pelo esquecimento, seja pela falta de ciência do usuário sobre a marcação das consultas.

Eu chegava a ponto de ligar para o paciente. Você sabia que amanhã você tem uma audiometria? (risos) Não, eu não fui avisado. Eu cansei. Não dá para fazer isso. Ou faço uma coisa ou eu faço outra. (Esperança, fonoaudióloga audiologista)

Destacamos uma análise do trecho acima: uma ligação do profissional com a criança, monitorando seus passos dentro do sistema, em um mix de responsabilidade / compromisso / preocupação. Essa ligação nos parece muito própria do que outros estudos apontam quando exploram pesquisas com crianças vivendo com condições crônicas complexas de saúde ${ }^{24-25}$. O fato de precisarem de cuidados específicos e especializados, as colocam ligadas por vezes desde o início da vida aos pontos mais complexos do sistema de saúde, qualificando-as simbolicamente e informalmente com os sobrenomes dos hospitais especializados que se tornam suas grandes referências. Tornam-se as crianças do Fernandes Figueira, do IPPMG, e assim por diante ${ }^{25}$. Esse sentimento de que a criança complexa pertence a um lugar complexo é retroalimentado pelos 
sentimentos de insegurança dos profissionais que a receberão, por exemplo, nos serviços locais de emergência, por queixas simples de saúde que elas também poderão vir a ter. A ideia da "minha criança”, ou da criança que foi sustentada pela hiperespecialização aí reside.

\section{A distância dos locais de consulta}

Além das dificuldades relacionadas ao tempo de espera, outro problema apontado nas entrevistas é a realização de encaminhamentos de crianças para unidades prestadoras muito distantes do bairro de residência. Este fator, por exemplo, dificulta especialmente o tratamento de crianças com doenças crônicas complexas de saúde que frequentam serviços de reabilitação ou de estimulação precoce. A distância as afeta não só pelo longo tempo de tratamento e frequência das consultas, mas também pelas dificuldades de locomoção no trajeto até os serviços. Logo, para que seja eficiente, se torna primordial que o tratamento destas crianças seja realizado perto de suas residências ${ }^{26}$.

Em revisão empreendida por Vilarins et al. ${ }^{1}$, há um destaque para o fato de os sistemas de regulação funcionarem como ferramentas para equidade, integralidade, acessibilidade e ajuste das ofertas assistenciais às necessidades do cidadão. No entanto, em passagem desse artigo, os autores questionam, mas não aprofundam uma crise entre o projeto proposto e a realidade:

Apesar dos avanços na forma de operacionalização da regulação assistencial, o processo de regulação em saúde ocorre ainda de forma bastante incipiente, requerendo aprimoramento em diversos aspectos. É importante dotar esse processo de instrumentos que garantam, de acordo com a necessidade de cada caso, a qualidade do atendimento aos pacientes e a alocação eficiente dos recursos médico -hospitalares. (p. 641) $)^{1}$

Um fator relacionado a esta questão apontado nas entrevistas é o cadastramento parcial de unidades e profissionais de saúde no SISREG, o que reduz ainda mais as opções de serviços e unidades prestadoras mais próximas à residência do usuário.

Se entra no SISREG, os fonoaudiólogos não estão no SISREG, muitos deles. (...) Então não tem como achar, né. Entendeu? O acesso é difícil. (Gratidão, fonoaudióloga de CMS)

Segundo Cavalcanti et al. ${ }^{23}$, um dos principais desafios da regulação assistencial pelo SISREG é a desorganização da rede de serviços que é gerada pela falta de pactuações de fluxos. Tal desorga- nização se reflete na desarticulação regional dos serviços e no desconhecimento da estruturação e fluxo da rede assistencial ${ }^{23}$.

Estudos internacionais ${ }^{26-27}$ apontam a importância da integração entre serviços baseados na comunidade e outros que operam nas diversas interfaces com processos de hospitalização e desospitalização, reabilitação, advocacy, de crianças com condições crônicas e complexas de saúde. Essas considerações só fortalecem nossa discussão de que dificuldades no estabelecimento de um fluxo de rede com acessos facilitados a essas crianças complexas podem não só comprometer seriamente seu crescimento, desenvolvimento, mas sua qualidade de vida e de suas famílias.

\section{Equívocos no processo de encaminhamento e o papel do NASF-AB}

$\mathrm{O}$ agendamento incorreto de consultas no SISREG também se mostrou um fator agravante na geração de dificuldades do acesso. Tal erro leva famílias a peregrinar pelas unidades de saúde buscando o serviço e/ou profissional que oferece o serviço compatível com a necessidade da criança. Neste sentido, alguns estudos sobre a atenção à saúde das crianças com condições crônicas complexas de saúde têm sinalizado a constante peregrinação destas crianças em busca de cuidados em saúde ${ }^{28-29}$.

Associada a esta questão comparece a informação de que muitos erros de marcação teriam ocorrido por divergências entre a nomenclatura utilizada pelo SISREG e àquela compartilhada entre os profissionais da assistência. Uma participante destaca que o BERA, exame solicitado por diversos profissionais da saúde e que afere o potencial aditivo, está referido no sistema como "Reabilitação em pacientes com perda auditiva", ou seja, um exame de diagnóstico está nomeado como serviço de tratamento. Esta divergência de nomenclatura foi apontada como um fator importante para a ocorrência de equívocos nos encaminhamentos, o que corrobora com o estudo de Cavalcanti et al. ${ }^{23}$ que reconhecem a existência de grande diversidade de nomenclatura das unidades de saúde e variedade de oferta de serviços relacionando-os à desorganização dos serviços de saúde.

Os participantes da pesquisa também referiram outro problema relacionado ao SISREG: a pouca clareza operacional do sistema. A nomenclatura utilizada para indicar as unidades e serviços prestadores não é autoexplicativa. Segundo algumas entrevistas, o regulador precisa explorar o sistema para descobrir como ele funciona. 
E essa questão da nomenclatura mesmo. Até a gente entender, um paciente com necessidade especial que a gente quer encaminhar para o NAIDI, você tem que colocar específico...é..serviço de reabilitação neurológica infantil. Isso a gente descobre futucando porque também nunca foi dito. (...) Então tem que ir descobrindo por tentativa e erro. E nisso quem sofre é o paciente. (Solidariedade, fonoaudióloga do NASF-AB)

Neste estudo, o profissional do Núcleo Ampliado de Saúde da Família e Atenção Básica (Nasf-AB) destacou-se como um importante ator na facilitação do acesso de usuários aos serviços da atenção especializada. No decorrer da pesquisa de campo, fomos instigados pela descoberta de uma estratégia utilizada por dois entrevistados específicos. Curiosamente, esses participantes não apresentaram queixas tão contundentes ao SISREG quando comparados aos demais entrevistados.

A particularidade destes profissionais estava na proximidade deles com o médico regulador da unidade onde atuam. A comunicação direta com o regulador permitia o direcionamento mais rápido e preciso das marcações de consultas das crianças assistidas por eles:

Olha...e como eu tenho acesso ao médico regulador isso me facilita muito, né. Eu já chego lá, e falo "Olha, regula aqui pra mim pra esse lugar porque eu já sei que aqui funciona bem. (Afeto, fonoaudióloga de PS).

Aí por whatsApp eu peço para uma médica reguladora:"Escuta, você pode regular?" Aí ela regula, aí eu consigo entregar na hora pro meu paciente. (...) tem vezes que eu não consigo (...)vários momentos eu consigo atender aquele paciente rapidinho e ele já sai com o dia e horário marcado. (Compromisso, fonoaudiólogo de CMS)

Entendendo que o NASF-AB possui um papel de apoiar a Estratégia Saúde Família (ESF) e que por isso tem uma posição estratégica junto aos reguladores nas unidades de atenção básica, mostrou-se importante ouvir estes profissionais para entender melhor a questão. O NASF-AB atua na Atenção Primária à Saúde (APS) e por isso tem acesso ao conhecimento do perfil da comunidade e do território. Através das entrevistas realizadas com duas fonoaudiólogas do NASF-AB que participam do grupo virtual, foi possível confirmar a importância do papel estratégico deste profissional também na qualificação do fluxo de usuários pela regulação de vagas através do SISREG ${ }^{30-32}$.

Para as fonoaudiólogas do NASF-AB que foram entrevistadas, falta aos reguladores o conhecimento específico das especialidades necessário para a realização adequada dos encaminhamentos. Neste sentido, o NASF-AB apresenta uma importante função de orientação e de apoio para a regulação das consultas relacionadas às especialidades, neste caso, à fonoaudiologia.

A fono do NASF é reguladora. É ela quem vai decidir pra onde aquele paciente vai. Então assim, é nossa função aqui dizer para o médico, pro enfermeiro, pra equipe pra onde tem que ser determinado paciente, porque ele não sabe (...) Ele abre, se eu descrever no SISREG pra ele "Ah! encaminha pra fono." Ele vai abrir e vai mandar pra uma pessoa que não tem nada a ver com linguagem. Se eu não chegar pra ele (...) e dizer "olha, aqui tem a fono de linguagem". Ele encaminha errado. (...) É uma coisa muito comum. (Empenho, fonoaudióloga do NASF-AB)

Nota-se ainda o papel desempenhado pelas fonoaudiólogas do NASF-AB como profissionais generalistas na APS. Elas realizam a triagem de demandas apresentadas pelos usuários do território e oferecem a assistência para os casos que consideram menos complexos. Essa ação mostrou-se uma estratégia interessante, visto que pode contribuir com a redução de sobrecarga dos ambulatórios especializados da atenção secundária. Nas entrevistas, várias estratégias foram relatadas neste sentido, tais como, a realização de grupos terapêuticos, atendimento mensais de orientação às famílias e também parcerias com as escolas. Segundo as entrevistadas, só os casos mais complexos são encaminhados por elas para a atenção especializada.

Reconhecemos a importância do papel matriciador deste profissional e sua posição estratégica como articulador e mediador dentro da rede de atenção à saúde. A existência de um vazio na intermediação entre os profissionais que atuam nos três níveis de atenção a saúde - primária, secundária e terciária - não permite que as especialidades funcionem de uma maneira clara, inclusive com relação ao fluxo de encaminhamentos. O NASF-AB é um ator central na intermediação entre a atenção básica e especializada ${ }^{33}$. Tal atuação favorece o aprimoramento do sistema de saúde para além do paradigma biomédico, em uma forma de organização de rede, evidenciando que a rede de atenção à saúde para funcionar precisa de algo que nos diga a respeito das entradas transversais.

As mudanças no cotidiano profissional com o início da regulação das vagas pelo SISREG ganhou destaque no estudo, e o reconhecimento de que o projeto é pertinente e bem idealizado. Ou seja, se as relações de familiaridade, das redes de con- 
fiança, amizade ${ }^{20}$ e conhecimento podem agilizar fluxos de encaminhamento, por outro lado elas deixam de fora quem por algum motivo está fora dessa lógica, enfraquecendo os ideais de equidade e universalidade do sistema de saúde, e a própria ideia de um Estado republicano, cuja base está o direito à saúde sem discriminação. Essa lógica não é exclusiva da área da saúde e, como assinala Mendonça ${ }^{34}$ em uma pesquisa sobre gestão do ensino público brasileiro, o Estado brasileiro constitui-se a partir de um modelo doméstico de relações sociais, onde predominam as vontades particulares mais que as ordenações impessoais que caracterizam o Estado burocrático (p. 96). Nesse sentido, a proposta da regulação que o SISREG atualiza, se vê obstaculizada não somente pelas falhas ainda presentes na própria operação do sistema, como pela cultura da informalidade, ou dos laços de referência, que remetem ao "quem indica".

Ao mesmo tempo, essa imagem de que algo se rompe nas interações diretas, mediadoras de laços e interações, quando o sistema de regulação é instituído, nos leva a refletir sobre as dimensões da cultura e símbolos que fazem sentido na vida das pessoas, que atravessam o cuidado. Portugal ${ }^{21}$ ao discutir redes sociais e seu papel nos cuidados de saúde, nos lembra que as redes de amizade e familiaridade oferecem aquilo que o Estado falha. E, portanto, os indivíduos buscam e conseguem através dos laços sociais aquilo que as relações de cidadania não garantem. O modelo da regulação, que o SISREG no município propõe, enfrenta não só seus problemas operacionais, mas também os impasses de enfrentar uma cultura em que interações diretas, relações de proximidade, indicações, oferecem aos que fazem parte dessas redes de benefícios.

O profissional de saúde que trabalha na ponta do sistema é um ator que se dedica à concretização do cuidado em saúde. Sua prática muitas vezes envolve a busca de soluções para as dificuldades encontradas no dia a dia profissional a fim de garantir a assistência à saúde dos seus pacientes ${ }^{35}$. Nesse caso, prevalece a dimensão das interações diretas, em que o compromisso do cuidado implica encaminhar e resolver, se possível com agilidade, e que podem contar com as redes informais de conhecimento e familiaridade a qual um sistema de regulação não contempla. Para convencer ou mobilizar mudanças nessa cultura, esses sistemas de regulação precisariam ser eficientes ao extremo para reafirmar que as relações de cidadania, mediadas pelo Estado, podem garantir acesso equânime e ágil para problemas de saúde que não podem esperar.
Muito embora as redes sociais de conhecimento e referências afetivas operem, elas não são suficientes para enfrentar ou produzir resoluções para as restrições de acesso. Isso porque questões de ordem operacional, infraestrutura, concursos públicos para absorção de profissionais, investimentos nas condições de trabalho, atualização profissional e salários, dentre tantos outros fatores, também operam como uma rede de efeitos iatrogênicos que comprometem o sistema de regulação, o acesso e o fluxo das crianças que demandam a intersetorialidade dos serviços de saúde, reabilitação, educação e garantia de direitos.

\section{Conclusão}

Neste estudo, a apreensão dos sentidos de fonoaudiólogos sobre as dificuldades de crianças com condições crônicas complexas de saúde em acessar a atenção especializada nos ajudou a refletir o lugar da fonoaudiologia como uma das especialidades que permite pensar as necessidades para essas crianças. Entrar nesta discussão pela fonoaudiologia foi uma forma tanto o de acessar os problemas mais gerais que as atingem quanto o de refletir sobre as demais especialidades que são muito acionadas por elas, em uma linha de cuidados para a saúde da criança na interface com os três níveis de atenção.

As principais dificuldades no acesso de crianças à rede especializada apontadas pelos entrevistados foram as barreiras nos fluxos dos encaminhamentos aos serviços, exatamente onde as interações pautadas no reconhecimento e familiaridade poderiam operar, com indicações pelas redes informais.

Identificamos que a regulação assistencial realizada via SISREG foi apontada como um agravante das dificuldades de acesso apresentadas por essas crianças. As dificuldades associadas à implantação do sistema de regulação agrupadas em três eixos principais: espera pela marcação das consultas, distância dos locais de consulta e erros de encaminhamento.

Além disso, neste estudo nos deparamos com a APS e o fonoaudiólogo do NASF-AB reconhecendo a importância deste profissional como um ator que ocupa um papel estratégico dentro do sistema de saúde enquanto principal matriciador no nível da atenção primária, que é a porta de entrada e coordenadora do cuidado dos usuários nos demais níveis de atenção. Neste sentido, o fonoaudiólogo do NASF-AB mostrou-se um importante apoiador e cooperador das redes de 
atenção, podendo melhor incluir e direcionar as crianças que referimos neste estudo.

De uma forma geral, o desafio está no estabelecimento de um sistema de regulação que de certa maneira extingue um modo de funcionar e de fazer diferente, referido a um modo de fazer baseado nas relações de cuidado, comprometidas com os ideais da resolução de problemas e encaminhamentos ágeis baseados muitas vezes nas redes de referências profissionais, de amizade e confiança.

\section{Colaboradores}

MF Silva realizou a pesquisa, analisou os resultados e redigiu o artigo. MCN Moreira orientou a pesquisa, a análise dos resultados, redigiu o artigo e realizou a revisão final do manuscrito. 


\section{Referências}

1. Vilarins GCM, Shimizu HE, Gutierrez MMU. A Regulação em Saúde: aspectos conceituais e operacionais. Saude Debate 2012; 36(95):640-647.

2. Conselho Nacional de Secretários de Saúde (CONASS). Programa de informação e apoio técnico às equipes gestoras estaduais do SUS. Brasília; 2007.

3. Cechinel C. Regulação em saúde: um diálogo com o princípio da equidade [dissertação]. Florianópolis: Universidade Federal de Santa Catarina; 2014.

4. Saffier IP, Silva MVCB. Consultas e exames na rede pública municipal do Rio de Janeiro via SISREG: desafios e alternativas de gestão para desafogar a demanda. Academus Revista Científica da Saúde 2017; 2(2):1-11.

5. Moreira MEL, Goldani MZ. A criança é o pai do homem: novos desafios para a área de saúde da criança. Cien Saude Colet 2010; 15(2):321-327.

6. Moreira MCN, Gomes R, Sá MRC. Doenças crônicas em crianças e adolescentes uma revisão bibliográfica. Cien Saude Colet 2014; 19(7):2083-2094.

7. Santos DCC, Campos D. Desenvolvimento motor - fundamentos para diagnóstico e intervenção. In: Moura-Ribeiro MVL, Gonçalves VMG. Neurologia do desenvolvimento da criança. Rio de Janeiro: Revinter; 2010. p. 288-307.

8. Feldman DE, Champagne F, Korner-Bitensky N, Meshefedjian G: Waiting time for rehabilitation services for children with physical disabilities. Child: Care, Health \& Development 2002; 28(5):351-358.

9. Nóbrega VM, Reichert APS, Viera CS, Collet N. Longitudinalidade e continuidade do cuidado à criança $\mathrm{e}$ ao adolescente com doença crônica. Escola Anna Nery 2015; 19(4):656-662.

10. Moreira MCN, Cunha CC, Mello A. Conversando sobre as crianças e os adolescentes com condições crônicas complexas na atenção primária: a atenção domiciliar gerando interfaces entre as especialidades e o cuidado no domicílio. In: Santos IS, Goldstein RA, organizadoras. Rede de pesquisa em Manguinhos: sociedade, gestores e pesquisadores em conexão com o SUS. São Paulo: Editora Hucitec; 2016. p.175-192.

11. Ferigollo JP, Kessler TM. Fisioterapia, fonoaudiologia e terapia ocupacional - prática interdisciplinar nos distúrbios da comunicação humana. Rev CEFAC 2017; 19(2):147-158.

12. Pichini FS, Rodrigues NGS, Ambrós TMB, Souza APR. Percepção da família e do terapeuta sobre a evolução de crianças em uma abordagem interdisciplinar de intervenção precoce. Rev CEFAC 2016; 18(1):55-66.

13. Madureira AF, Moreira MCN, Sá MC. A Fisioterapia para Novos Atores: Disputas e Inovações para Atenção às Crianças Cronicamente Adoecidas. Cien Saude Colet; [periódico na internet] 2017 Set [Citado em $18 \mathrm{dez}$ 2018]. Disponível em: http://www.cienciaesaudecoletiva.com.br/artigos/a-fisioterapia-para-novos-atoresdisputas-e-inovacoes-para-atencao-as-criancas-cronicamente-adoecidas $/ 16398$ ? id $=16398$.

14. Silva MF. Sobre redes virtuais sentidos e ações tecidas: quais saídas para a peregrinação por acesso à atenção especializada em uma linha de cuidados para a saúde da criança? [dissertação]. Rio de Janeiro: Instituto Nacional de Saúde da Mulher, da Criança e do Adolescente Fernandes Figueira; 2018.
15. Iriart JAB, Silva LAV. As tecnologias da informação e comunicação e novas formas de sociabilidade: possibilidades e desafios para o campo da saúde coletiva. Cad Saude Publica 2015; 31(11):2253-2254.

16. Costa R. Por um novo conceito de comunidade: redes sociais, comunidades pessoais, inteligência coletiva. Interface (Botucatu) 2005; 9(17):235-248.

17. Ayres M, JC Ribeiro. A representação de si em interações sociais mediadas por instant messengers: o caso whatsapp. Intercom 2015;1-15.

18. Gomes R, Souza ERD, Minayo MCDS, Malaquias JV, Silva CFRD. Organização, processamento, análise e interpretação de dados: o desafio da triangulação. In: Minayo MCS, Assis SG, Souza ER, organizadoras. Avaliação por triangulação de métodos - Abordagem de programas sociais. Rio de Janeiro: Fiocruz; 2005. p. 185-222.

19. Barnes JA. Redes sociais e processo político. In: Feldman-Bianco B, organizadora. Antropologia das sociedades complexas contemporâneas: métodos. São Paulo: Global; 1987. p.159-191.

20. Boissevain J. Apresentando "Amigos de amigos: redes sociais manipuladores e coalisões" In: Feldman-Bianco B, organizadora. Antropologia das sociedades complexas contemporâneas: métodos. São Paulo: Global; 1987. p.195-223.

21. Portugal S. "Quem tem amigos tem saúde": O papel das redes sociais no acesso aos cuidados de saúde. In: Simpósio "Família, redes sociais e saúde"; 2005; Hamburgo. p. 1-23.

22. Portugal S. Quanto vale o capital social: o papel das redes informais na provisão de recursos. In: Fontes B, Martins PH. Redes, práticas associativas e gestão pública. Recife: UFPE; 2006. p. 51-74.

23. Cavalcanti AG. A regulação assistencial no Sistema Único de Saúde: o caso da Central de Regulação de Cuiabál MT [dissertação]. Cuiabá: Universidade Federal de Mato Grosso; 2011.

24. Moreira MCN, Albernaz LV, Sá MRC, Correia RF, Tanabe RF. Recomendações para uma linha de cuidados para crianças e adolescentes com condições crônicas complexas de saúde. Cad Saude Publica 2017; 33(11):1-13.

25. Moreira MCN, Moura EC, Gomes R, Menezes LA, Sá MRC, Correia RF, et al. Diagnóstico das condições crônicas em pediatria no Instituto Fernandes Figueira/FIOCRUZ: retrato da morbidade hospitalar e linhas de cuidados. Relatório de pesquisa. Rio de Janeiro: Fundação Oswaldo Cruz; 2016.

26. Kuo DZ, Houtrow AJ. Council on Children With Disabilities. Recognition and management of medical complexity. Pediatrics 2016; 138(6).

27. Cohen E, Kuo DZ, Agrawal R, Berry JG, Bhagat SK, Simon TD, Srivastava R. Children with medical complexity: an emerging population for clinical and research initiatives. Pediatrics 2011; 127(3):529-538.

28. Pereira TIAFA. A Estratégia de Saúde da Família na garantia do acesso da criança com deficiência à rede de atenção [dissertação]. Rio de Janeiro: Fundação Oswaldo Cruz; 2016

29. Rosário SSDD, Fernandes APNDL, Batista FWB, Monteiro AI. Acessibilidade de crianças com deficiência aos serviços de saúde na atenção primária. Rev eletrônica enferm 2013; 15(3):740-746. 
30. Lima TFP, Acioli RML. A inserção da fonoaudiologia na atenção primária do sistema único de saúde. In: Silva VL, Lima MLLT, Lima TFP, Advíncula KP. A prática fonoaudiológica na atenção primária à saúde. São José dos Campos: Pulso editorial, 2013. p. 25-42.

31. Lima MLLT, Teixeira CF, Macedo CA, Barboza CMN. Núcleo de Apoio à Saúde da Família: aspectos normativos e panorama no Brasil. In: Silva VL, Lima MLLT, Lima TFP, Advíncula KP. A prática fonoaudiológica na atenção primária à saúde. São José dos Campos: Pulso editorial, 2013. p. 43-54.

32. Brasil. Ministério da Saúde (MS). O Nasf nas Redes: Integração entre Serviços da Rede de Atenção à Saúde e Articulação Intersetorial. In: Cadernos de atenção básica. Nucleo de apoio à saúde da família - volume 1: ferramentas para gestão e para o trabalho cotidiano. Brasília: MS; 2014.

33. Sampaio J, Sousa CSM, Marcolino EC, Magalhães FC, Souza FF, Rocha AMO, Neto AAS, Sobrinho GDO. O NASF como dispositivo da gestão: limites e possibilidades. Revista Brasileira de Ciências da Saúde 2012; 16(3):317-324.

34. Mendonça EF. Estado patrimonial e gestão democrática do ensino público no Brasil. Educ Soc 2001; 22(75).84-108.

35. Martins PH, Cuentro AC. Os profissionais de saúde como mediadores individuais: a resolução da demanda ilimitada por cidadania pelo mecanismo do duplo registro. In: Pinheiro R, Martins PH, organizadores. Usuários, redes sociais, mediações e integralidade em saúde. Rio de Janeiro: UERJ/ IMS/ LAPPIS; 2011. p. 139-147.

Artigo apresentado em 19/12/2018

Aprovado em 30/06/2019

Versão final apresentada em 02/07/2019

Editores-chefes: Romeu Gomes, Antônio Augusto Moura da Silva 